\title{
RNAseq-based Transcriptome Analysis of Burkholderia glumae Quorum Sensing
}

\author{
Sunyoung Kim ${ }^{1}$, Jungwook Park ${ }^{1}$, Ji Hyeon Kim ${ }^{1}$, Jongyun Lee ${ }^{1}$, Bongjun Bang ${ }^{1}$, Ingyu Hwang ${ }^{2}$ and \\ Young-Su Seo ${ }^{1 *}$ \\ ${ }^{1}$ Department of Microbiology, Pusan National University, Busan 609-735, Korea \\ ${ }^{2}$ Department of Agricultural Biotechnology, Seoul National University, Seoul 151-921, Korea \\ (Received on April 29, 2013; Revised on May 16, 2013; Accepted on May 16, 2013)
}

\begin{abstract}
Burkholderia glumae causes rice grain rot and sheath rot by producing toxoflavin, the expression of which is regulated by quorum sensing (QS). The QS systems of $B$. glumae rely on $N$-octanoyl homoserine lactone, synthesized by TofI and its cognate receptor TofR, to activate the genes for toxoflavin biosynthesis and an IclR-type transcriptional regulator gene, qsmR. To understand genome-wide transcriptional profiling of QS signaling, we employed RNAseq of the wild-type B. glumae BGR1 with QS-defective mutant, BGS2 (BGR1 tofI:: $\Omega$ ) and QS-dependent transcriptional regulator mutant, BGS9

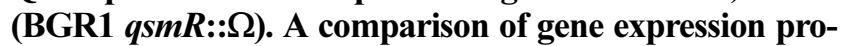
filing among the wild-type BGR1 and the two mutants before and after QS onset as well as gene ontology (GO) enrichment analysis from differential expressed genes (DEGs) revealed that genes involved in motility were highly enriched in TofI-dependent DEGs, whereas genes for transport and DNA polymerase were highly enriched in QsmR-dependent DEGs. Further, a combination of pathways with these DEGs and phenotype analysis of mutants pointed to a couple of metabolic processes, which are dependent on QS in B. glumae, that were directly or indirectly related with bacterial motility. The consistency of observed bacterial phenotypes with GOs or metabolic pathways in QS-regulated genes implied that integration RNAseq with GO enrichment or pathways would be useful to study bacterial physiology and phenotypes.
\end{abstract}

Keywords : Burkholderia glumae, gene ontology enrichment, pathway, quorum sensing, RNAseq

The bacterium Burkholderia glumae is a causative agent of rice grain rot and bacterial wilt on various field crops including pepper, tomato, and eggplant (Kim et al., 2010; Nandakumar et al., 2009). The occurrence and disease damages due to $B$. glumae become a serious problem in many rice-growing countries at the flowering stages in high

\footnotetext{
*Corresponding author.

Phone)+82-51-510-2267, FAX) +82-51-514-1778

E-mail)yseo2011@pusan.ac.kr
}

humid and high temperature conditions. Although the development of resistance varieties or chemical treatments to reduce the damage caused by B. glumae is in progress, several virulence factors have been identified. B. glumae produces toxoflavin, a host-nonspecific phytotoxin, which is a very effective electron carrier and generates reactive oxygen species (ROS) such as hydrogen peroxide and acts as a major virulence factor for B. glumae in rice (Sato et al., 1989). Interestingly, B. glumae cells can develop a system to detoxify ROS generated by either toxoflavin or host defense at the infection sites. Recently, B. glumae KatG was demonstrated to function as a catalase both to detoxify oxidative stress as well to function as a virulence factor (Chun et al., 2009). Bacterial motility driven by flagella and lipase production is necessary for the pathogenicity of $B$. glumae (Devescovi et al., 2007; Kim et al., 2007). All these virulence factors mentioned above are regulated by celldensity-dependent manner of quorum sensing (QS).

Bacterial QS is a cell-to-cell communication using chemical signals to monitor bacterial environment and regulate many bacterial behaviors. The most well-known chemical signals are $N$-acylhomoserine lactones (AHLs). In AHL QS system, the regulatory circuit is made up of a signal producer named the LuxI and a cognate receptor-regulatory protein designated as LuxR (Fuqua et al., 1994). Bacterial cells secrete and respond to AHL as an autoinducer to sense and respond to events, leading to cell division, production of toxin and biofilm, and controlling of bacterial colonization and pathogenesis in the host.

Using a TofI-TofR circuit in QS, B. glumae QS performs its action. TofI, a LuxI-family protein, synthesizes the autoinducer, which is recognized by TofR, a LuxR-family protein. TofR-C8-HSL triggers downstream events of QS in $B$. glumae. For example, TofR-C8-HSL activates the expression of an IclR-type transcriptional regulator gene, $q s m R$, which is a master regulator, and then QsmR activates the downstream regulator for flagellar gene expression. The biosynthesis of toxoflavin is tightly regulated by QS through activation of the expression of the tox $A B C D E$ gene cluster for toxoflavin biosynthesis and tox $F G H I$ gene cluster for its 
transport (Kim et al., 2009). B. glumae KatG-mediated detoxifying ROS produced either by toxoflvin or cellular stress response is also regulated by QS.

To understand genome-wide transcriptional profiling of QS signaling, we employed RNAseq of the wild-type $B$. glumae BGR1 with QS-defective mutant, BGS2 (BGR1 tof $I: \Omega$ ) and QS-dependent transcriptional regulator mutant,

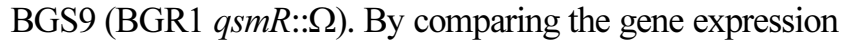
profiling among the wild-type BGR1 and the two mutants before ( $6 \mathrm{~h}$ LB liquid culture) and after (10 h LB liquid culture) the onset of QS, 1133 TofI-dependent and 840 QsmR-dependent genes were identified. Enrichment of gene ontology (GO) revealed the unique and shared signaling cascades between TofI- and QsmR-dependent QS. TofI- or QsmR-dependent genes highly enrich GO biological processes that involve regulation of transcription, response to (oxidative) stress, and transport. GO biological processes that consist of protein secretion and folding are specifically enriched in only QsmR-dependent genes.

Consistent with a previous report (Kim et al., 2007) and observed phenotypes from this study, motility-related genes are positively up-regulated in a QS-dependent manner. Interestingly, motility-related GO terms including flagellum assembly and motility are highly enriched in TofI-dependent genes but not in QsmR-dependent ones, though QsmRdependent flhDC regulation in B. glumae bacterial motility was reported (Kim et al., 2007), suggesting that TofI can regulate motility not only through QsmR-dependent flhDC regulation (Kim et al., 2007) but also through other signal- ing pathways. This notion was supported by the finding that one of the amino acid degradation pathways, arginine degradation II (AST pathway) and fatty acid $\beta$-oxidation I regulated by QS, was indirectly or directly involved in bacterial swarming motility. A combination of QS-regulated genes with GO enrichment and pathway analysis can provide an efficient approach to dissect signaling cascades and phenotype analysis in B. glumae.

\section{Materials and Methods}

Bacterial strains. All B. glumae strains used in this study are derivatives of the wild-type strain BGR1. BGS2 and BGS9 are derivatives of BGR1 tof $:: \Omega$ and BGR1 qsmR:: $\Omega$ respectively (Kim et al., 2004, 2007).

RNAseq data. We downloaded RNAseq raw data (GSE36485) previously deposited in gene expression omnibus (GEO) (http:/www.ncbi.nih.gov/geo/). The relative transcript abundance was measured in reads per kilobase of exon per million mapped reads (RPKM) (Mortazavi et al., 2008). $\log _{2}$ ratio of RPKM values were used to identify differential expressed genes.

RNA extraction. $B$. glumae BGR1, BGS2 (BGR1 tofI::S), and BGS9 (BGR1 qsmR:: $\Omega$ ) were grown in LB medium (Difco) at $37{ }^{\circ} \mathrm{C}$ with continuous shaking either for $6 \mathrm{~h}$ or 10 h. Total RNA was extracted by the RNeasy Midi Kit (Qiagen, Valencia, CA) following the manufacturer's proto-

Table 1. Primers used for quantitative real-time PCR to validate expression patterns of RNAseq data

\begin{tabular}{|c|c|c|}
\hline Locus ID & Gene function & $\begin{array}{l}\text { Sequence } \\
\left(5^{\prime} \text { to } 3^{\prime}\right)\end{array}$ \\
\hline bglu_1g13500* & ATP-dependent Clp protease proteolytic subunit & $\begin{array}{l}\text { F: TGATCAATCGCGCTGAATTGCTGG } \\
\text { R: ACAACTGCGGTAGCTGCAATGAAC }\end{array}$ \\
\hline bglu_1g02620 & 30S ribosomal protein $\mathrm{S} 19$ & $\begin{array}{l}\text { F: GACGCCCATTTGCTGAAGAAGGTT } \\
\text { R: ATGAAGTCCGGCAGAATCGTCGAA }\end{array}$ \\
\hline bglu_1g10680 & acylhomoserine lactone dependent transcriptional activator & $\begin{array}{l}\text { F: TTCACAGCAGGATCAATGCGAAGC } \\
\text { R: AATATCGGCGATCTCGCTGTACGA }\end{array}$ \\
\hline bglu_2g09850 & hypothetical protein & $\begin{array}{l}\text { F: TCATGTCGGAATTCCACCCGTTCA } \\
\text { R: TTGCGGCACCGAGAGAAACACTT }\end{array}$ \\
\hline bglu_2g11090 & type VI secretion system effector & $\begin{array}{l}\text { F: AAGCCGATTACGCTCACGAAGGAA } \\
\text { R: TCTTGACGTTGTCGAGCTTCGTGT }\end{array}$ \\
\hline bglu_2g18750 & aldehyde dehydrogenase & $\begin{array}{l}\text { F: GCCGATCCTCTACGTGCTGAAGTA } \\
\text { R: AGATCGGTCGTAAACACGCACGAC }\end{array}$ \\
\hline bglu_2g18780 & transferase & $\begin{array}{l}\text { F: TACAGCCTGGTCGGCATTCA } \\
\text { R: GCATAGAACTCGATGTTGCCGC }\end{array}$ \\
\hline bglu_2g11300 & acyl-CoA dehydrogenase & $\begin{array}{l}\text { F: ACATGGTGGCGATGGAGGAAATCT } \\
\text { R: ATGGATCTGGTTGACGCAGAGATTCG }\end{array}$ \\
\hline bglu_2g17540 & MutT/nudix family protein & $\begin{array}{l}\text { F: ACTACGTGCTGATCGCGTTGCTGT } \\
\text { R: TCGGAATGATTGATCTCGTGGGCA }\end{array}$ \\
\hline
\end{tabular}

\footnotetext{
*reference gene
} 
col. Total RNA was treated with RNase-free DNase (Invitrogen, Carlsbad, USA).

\section{Verification of expression patterns from RNAseq result} using quantitative real-time PCR. To validate expression patterns from RNAseq analysis, cDNA was generated by using SuperScript ${ }^{\mathrm{TM}}$ III First-Strand Kit (Invitrogen, Carlsbad, USA) with random hexamer. DNase-treated total RNA $(2-2.5 \mu \mathrm{g})$ was used as a starting material to generate cDNA by following the manufacture's protocol. The synthesized cDNA samples were diluted to $15-20 \mathrm{ng} / \mu \mathrm{l}$ using DEPCtreated water and were measured by Nano Drop 2000 (Thermo scientific, Wilmington, USA). In each reaction, 5 $\mu l$ of template was used. cDNA templates were mixed with QuantiTect SYBR Green PCR Kit (Qiagen, Valencia, USA) and specific primers as listed in Table 1. Each reaction included initial heating for $10 \mathrm{~min}$ at $95^{\circ} \mathrm{C}$, followed by 40 cycles of PCR $\left(95^{\circ} \mathrm{C}, 10 \mathrm{~s} ; 55-60{ }^{\circ} \mathrm{C}, 15 \mathrm{~s} ; 72^{\circ} \mathrm{C} 20 \mathrm{~s}\right)$. The relative transcription levels of the isotigs were calculated using comparative quantification by rotor-gene $\mathrm{Q}$ (Qiagen, Valencia, USA). The relative transcription levels were normalized by reference gene and compared with expression levels of RNAseq.

DNA construction and mutagenesis. General and standard techniques were used for DNA manipulation, cloning, and restriction digestion, and agarose gel electrophoresis (Sambrook et al., 1989). Single crossover recombination was used for mutagenesis of BGR1. For mutagenesis, the part of the astC (bglu_1g10100) was amplified and inserted into pVIK112. To insert the part of the target gene to pVIK112, EcoRI sequence was added to forward primer and $K p n I$ sequence was added to reverse primer; forward primer sequence: 5'-AAAAAGAATTCAACGGCTACAC CAACGA-3' and reverse primer sequence: 5'-AAGGTAC CCGAAGCCGTCCGAATACT-3'. After PCR and electrophoresis, the amplified fragment, taken from the location of the approximately 200 bp, was cleaved by EcoRI and KpnI, and pVIK112 was also cleaved by EcoRI and KpnI. The digested insert and vector were ligated by ligase and transformed into DH5 $\alpha \lambda$ pir competent cell. Transformed cells were cultured overnight in kanamycin medium $(50 \mu \mathrm{g} / \mathrm{ml})$. To identify proper ligation, we extracted the plasmid DNA from the cell and performed enzyme-cut and PCR. Properly combined plasmid DNA was transformed into S17-1 $\lambda$ pir and cloned. S17-1 $\lambda$ pir was mated with BGR1 and selected by LB medium containing rifampicin $(100 \mu \mathrm{g} / \mathrm{ml})$ and kanamycin $(100 \mu \mathrm{g} / \mathrm{ml})$. Colonies that grew on the rifampicin and kanamycin plates were collected and confirmed as insertional mutants using PCR with forward primer (5'ACGTGATGGTGCCGGTGTTC-3'), which is the upstream sequence of target gene, and reverse primer (5'-GGGGATG
TGCTGCAAGGCG-3'), which is internal sequence of pVIK112 vector. For pstA (bglu_1g11470) and fadE (bglu_ $2 \mathrm{~g} 06260)$ mutagenesis, we followed the same procedures as described in astC mutagenesis above. To insert the part of the pstA to pVIK112, EcoRI sequence was added to forward primer and $K p n \mathrm{I}$ sequence was added to reverse primer; forward primer sequence: 5'-AAAAAGAATTCG TCTACCTGGCCGAATAC-3' and reverse primer sequence: 5'-AAGGTACCCAGGTTCAGCATGTTCTC-3'. For confirmation of correct mutant colonies after mutagenesis, we used PCR with forward primer (5'-CAATGCCGTCGCG CTGA-3') and reverse primer (5'-GGGGATGTGCTGCAA GGCG-3'). In case of fadE mutagenesis, EcoRI sequence was added to forward primer and $K p n I$ sequence was added to reverse primer; forward primer sequence: 5'-AAAAGA ATTCGCACCCAGTTCGGCAAG-3' and reverse primer sequence: 5'-AAGGTACCTAGTCGGCCAGATAGCCAT AG-3'. For confirmation of correct mutant colonies after mutagenesis, we used PCR with forward primer (5'-TGCG AGATTCCCGAGGAGAA-3') and reverse primer (5'-GGGGATGTGCTGCAAGGCG-3').

Gene ontology enrichment. GO terms were downloaded from VertiGO database of MicrosOnline (http://www.micro besonline.org). The significance of enrichment for total of GO terms was calculated by Fisher's exact test, and the obtained p-values were adjusted for multiple hypothesis testing by q-value (Storey and Tibshirani, 2003).

Pathway analysis. For constructing pathway database, we used python code script and 'WGET' program of LINUX operating system. Pathway database was downloaded at the 'KEGG': Kyoto Encyclopedia of Genes and Genomes (http://www.genome.jp/kegg/) or Biocyc (http://biocyc.org/). Either pathway was combined with differential expressed genes from RNA-sequencing data.

Swarming (motility) assays. Swarming assays were performed at $37{ }^{\circ} \mathrm{C}$ on LB agar plates that contained $0.5 \%$ Bacto agar (Difco). Each strain was incubated at $37^{\circ} \mathrm{C}$ for $12-14 \mathrm{~h}$ with shaking after inoculated into $2 \mathrm{ml}$ of LB broth and then subcultured at $37^{\circ} \mathrm{C}$ for $4-5 \mathrm{~h}$ in $2 \mathrm{ml} \mathrm{LB}$ broth. To inoculate on LB agar medium for swarming assays, cultured cells of strain were centrifuged, washed twice with fresh LB broth, and resuspended in $100 \mu$ of LB broth. An aliquot $(2 \mu \mathrm{l})$ of each cell suspension was dropped onto LB agar medium and the assay plates were incubated for $24 \mathrm{~h}$.

\section{Results}

Identification of TofI- and QsmR-regulated genes using RNAseq. To demonstrate global regulatory functions of 


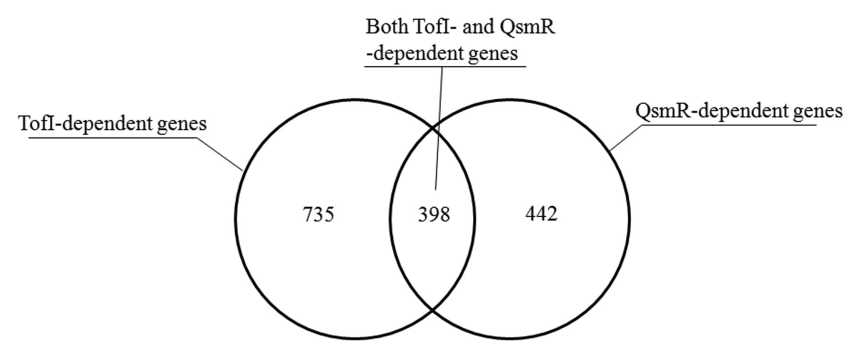

Fig. 1. Unique and common TofI- and QsmR-dependent genes. Differential expressed genes were selected from twofold changes of $\log _{2}$ ratio of wild-type/mutant RPKM values. 735,442 , and 398 are Tofl-, QsmR-, and both dependent genes, respectively.

QS, transcriptional profiling from RNAseq data was analyzed. We compared two QS mutants [BGS2 (BGR1 tofI:: $\Omega$ ) and BGS9 (BGR1 qsmR::S)] with wild-type BGR1 before and after the onset of QS. Relative quantification of sequenced RNAs in each RNAseq was measured by reads per kilobase of exon per million mapped reads (RPKM) (Mortazavi et al., 2008). To identify TofI- and QsmRregulated genes, differential expressed genes (DEGs) were selected using twofold changes of $\log _{2}$ ratio of (BGR1/ BGS2) and (BGR1/BGS9) RPKM values, respectively, as the criterion. Based on this criterion, 735 Tofl- and 442 QsmR-dependent genes were identified. In addition, 398 genes were both Tofl- and QsmR-regulated at the transcriptional level (Fig. 1).

Validation of the RNAseq expression patterns by quantitative real-time PCR (qPCR). Eight of the QS-regulated genes were randomly selected to validate RNAseq results and qPCR was carried out with gene-specific primers (listed in Table 1). RNA samples extracted from two additional biological replicate sets of cultures were used as templates for qPCR. The values were average of two biological replicates (Fig. 2). According to the results from 24 qPCR sets including the reference gene (blgu_1g13500), the trends of expression patterns of QS-regulated genes from RNAseq analysis were in good accordance with the expression levels by $\mathrm{qPCR}$ although the altitude of fold change is different due to sensitivity of each technique (Fig. 2).

Overview of TofI- and QsmR-dependent genes based on GO enrichment analysis implies common and unique functional categories. Among 735 TofI- and 442 QsmRdependent genes as well as 398 genes that were both TofI-
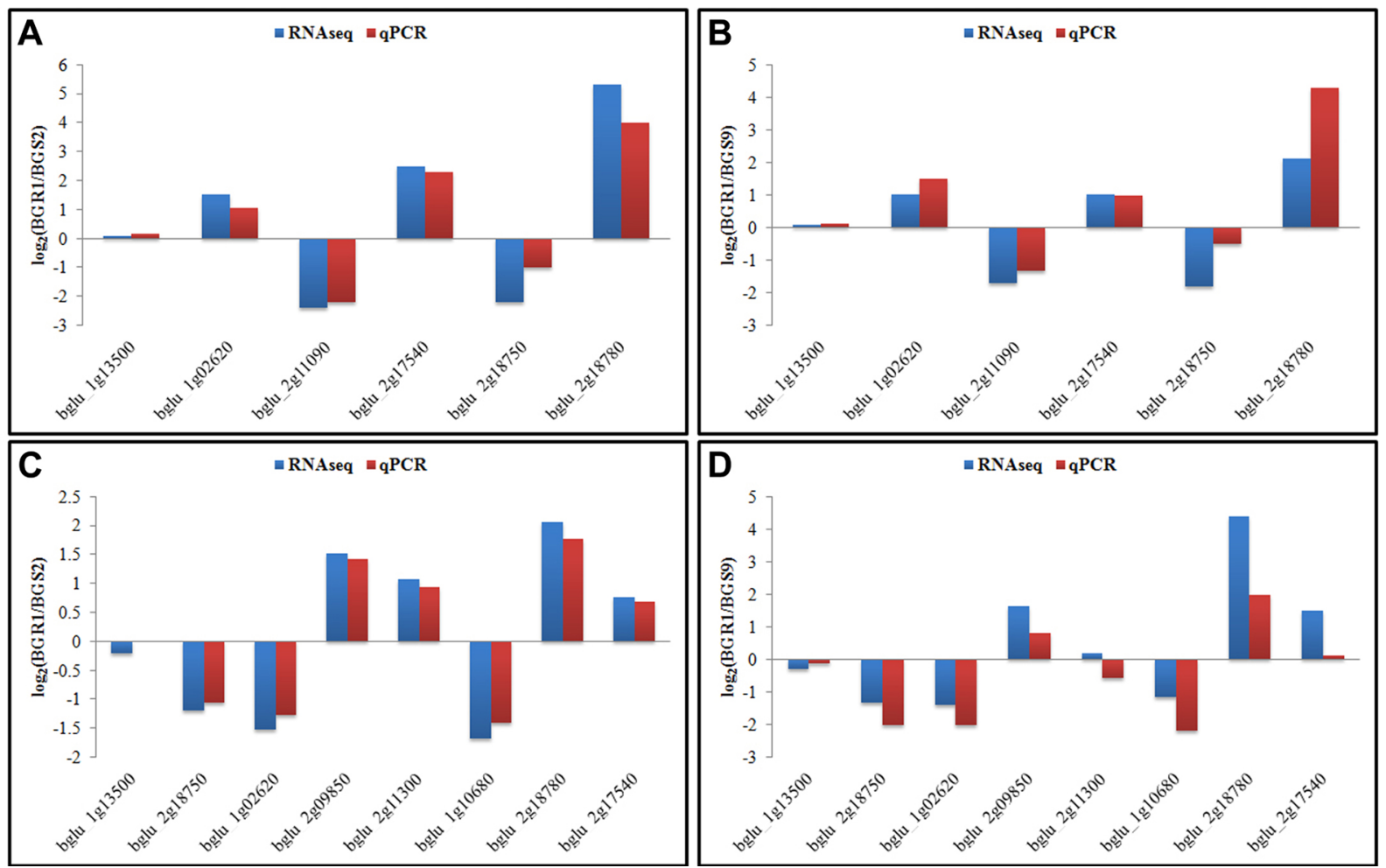

Fig. 2. Validation of RNAseq-based expression patterns using quantitative RT-PCR (qPCR). The expression patterns of randomly selected genes were analyzed by qPCR to validate RNAseq results. (A) Fold differences of BGR1 to BGS2 before quorum sensing (QS) onset. (B) Fold differences of BGR1 to BGS9 before QS onset. (C) Fold differences of BGR1 to BGS2 after QS onset. (D) Fold differences of BGR1 to BGS9 after QS onset. Each fold difference was calculated by qPCR using comparative quantification method, and $\log _{2}$ ratio of obtained values was compared with $\log _{2}$ ratio of (BGR1/BGS2) and (BGR1/BGS9) RPKM values. 
A

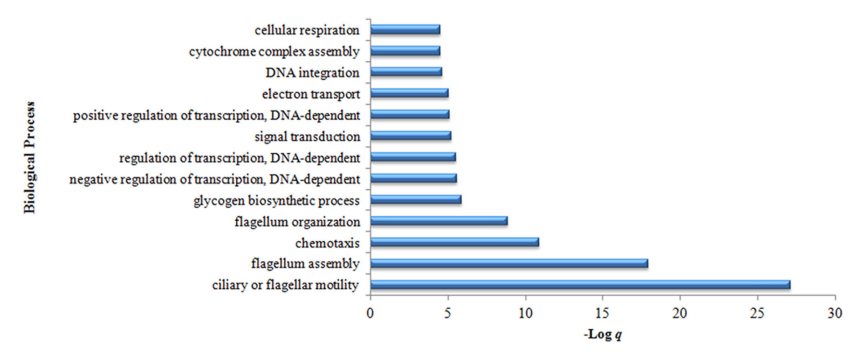

B

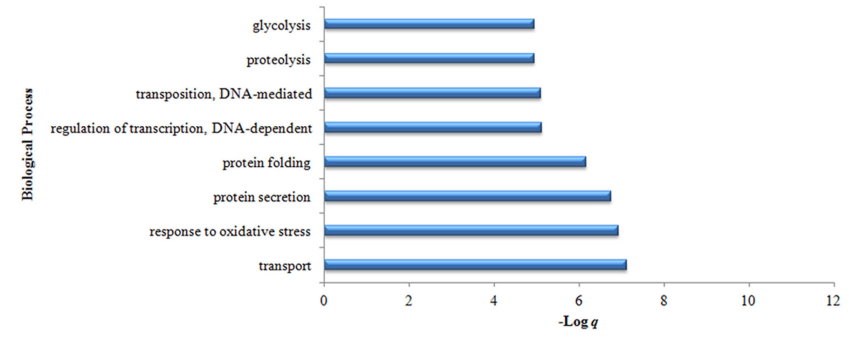

C

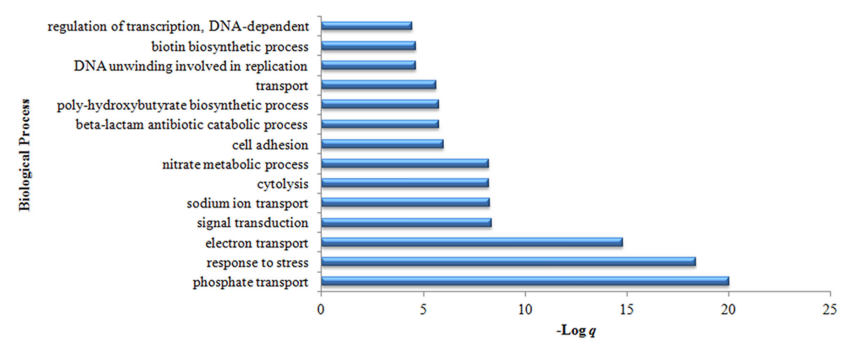

Fig. 3. Enrichment of gene ontology (GO) biological processes among differential expressed genes (DEGs). (A) Tofl-dependent DEGs; (B) QsmR-dependent DEGs; (C) both Tofl and QsmRdependent DEGs. The significance of enrichment was calculated by Fisher's exact test, and the obtained $p$-values were adjusted for multiple hypothesis testing by $q$-value (Storey and Tibshirani, 2003). GO biological process terms were enriched by $q<0.05$ (represented as $-\log (q)$ in the bar graph).

and QsmR-regulated, genes were grouped based on $\mathrm{GO}$ of MicrobesOnline (http://www.microbesonline.org). From GO analysis of TofI- and QsmR-dependent genes, 13 and 8 of $443 \mathrm{GO}$ biological process terms were enriched by $q<0.05$ (represented as $-\log (q)$ in the bar graph, Fig. 3A and 3B), respectively. Fourteen GO biological process terms were enriched in both TofI/QsmR-dependent genes by $q<0.05$ (Fig. 3C). Although a certain GO such as transcriptionrelated biological process was commonly enriched in both Tofl- and QsmR-dependent genes, a couple of GO terms were enriched independently. For example, motility-related GO biological processes including flagellar motility, assembly, and organization are highly enriched in Toff-dependent genes. However, GO terms presenting with protein secretion/ folding and response to oxidative stress were enriched in
QsmR-dependent genes.

In GO molecular function with the same criteria $(q<$ $0.05), 16,12$, and 20 of $993 \mathrm{GO}$ molecular function terms were enriched in TofI-, QsmR-, and TofI/QsmR-dependent genes, respectively. Among the GO terms, motor activity and structural molecular activity in TofI-dependent genes were highly enriched, whereas DNA-directed DNA polymerase activity was highly enriched in QsmR-dependent genes. Further, higher enrichment of GO with cofactor binding and phosphate transmembrane transporter activity was observed in Tofl/QsmR-dependent genes.

In GO cellular component with the same criteria $(q<$ $0.05), 6,3$, and 4 of 94 GO terms were enriched in Tofl-, QsmR-, and Toft/QsmR-dependent genes, respectively. Among the GO terms, flagellum-related GO was the highest enriched in Tofl-dependent genes, whereas intracellular GO was the highest enriched in QsmR-dependent genes. Interestingly, enzyme complexes such as nitrate reductase and acetyl-CoA carboxylase complex were highly enriched in TofI/QsmR-dependent genes.

Characterization and analyses of QS-dependent pathways. To understand the QS-dependent pathway, we merged pathways into QS-regulated genes from RNAseq analysis. One of major phenotypic defects in B. glumae QS mutants was a swarming motility (Fig. 4A-C). Both BGS2 and BGS9 are defective in motility. Sometimes, BGS9 showed a slight swarming motility, whereas BGS2 exhibited complete defects in motility (data not shown). This leads to the observation that swarming motility was more tightly regulated by TofI rather than QsmR. In addition, TofIdependent genes showed highly enriched GO motilityrelated terms (Fig. 3). Thus, we further analyzed the genes involved in motility from RNAseq data. Interestingly, all the 35 genes involved in flagellar assembly pathways downloaded from KEGG pathway database were highly upregulated after QS onset when genes of wild type were compared with those of BGS2 (Table 2 and Fig. 4D) under the criteria of twofold changes. However, only eight genes were highly up-regulated in QsmR-dependent genes after QS onset with the same criteria.

By contrast, genes involved in ribosome pathway were highly down-regulated in a QS-dependent manner. Among the 55 genes downloaded from KEGG pathway, all genes involved in ribosome pathway with a few exceptions were down-regulated from both Tofl- and QsmR-dependent RNAseq experiment (Table 3). As regards metabolic pathways, we also observed interesting gene expression patterns in arginine degradation II pathway (AST pathway). All genes involved in AST pathway and several key genes involved in fatty acid $\beta$-oxidation I were up-regulated after the onset of QS, whereas these genes were down-regulated before the 


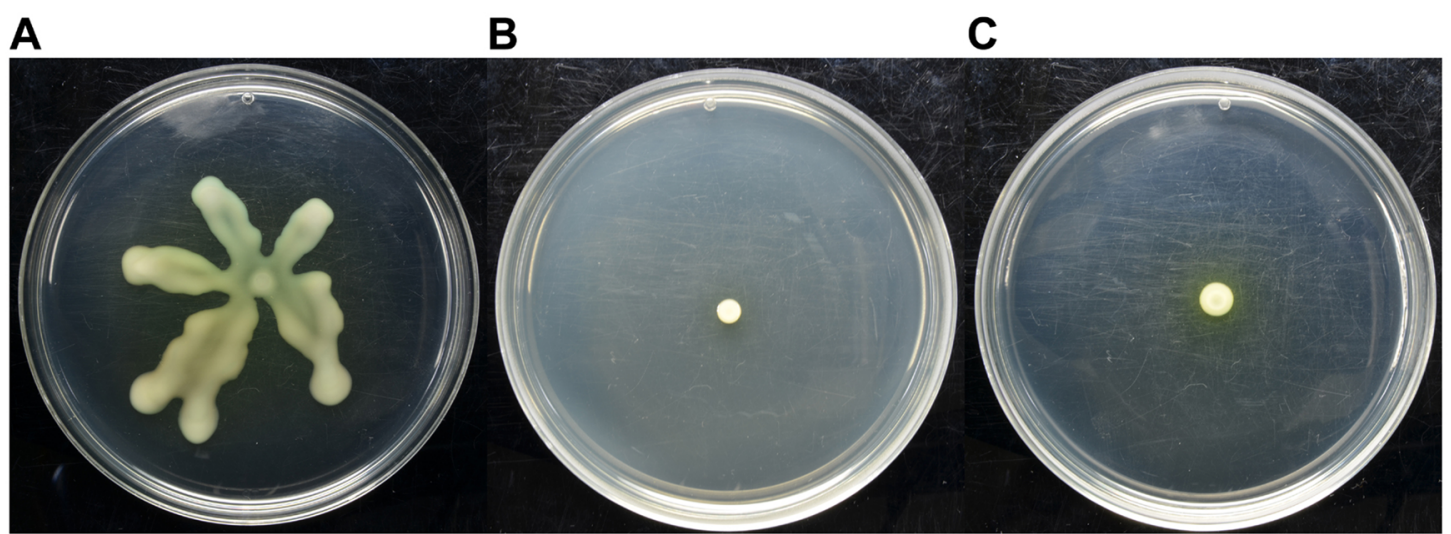

D
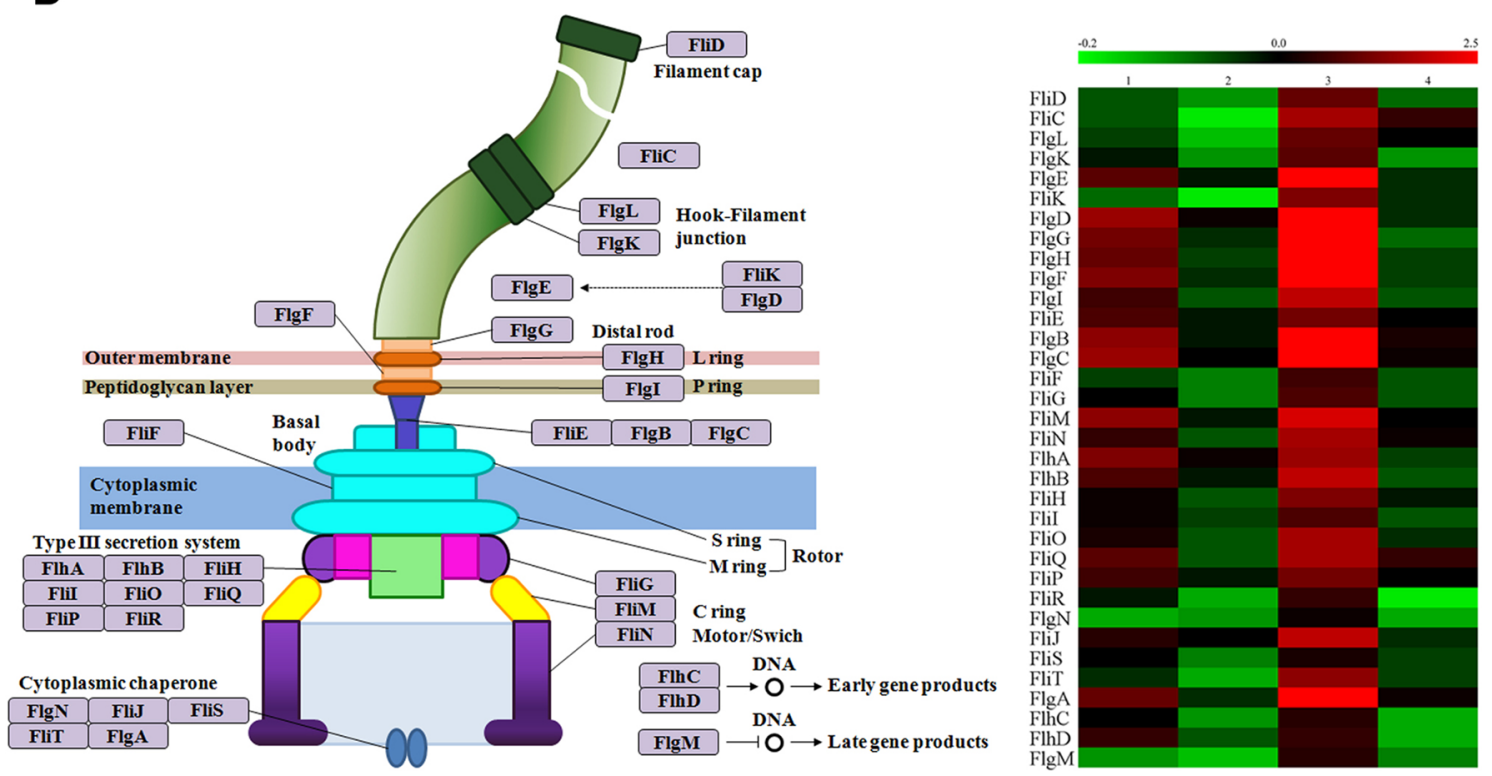

Fig. 4. Quorum sensing (QS)-dependent motility phenotypes and expression profiling of genes involved in flagellar assembly. (A-C) Swarming motility assays of wild-type BGR1, BGS2, and BGS9, respectively. An aliquot ( $2 \mu \mathrm{l})$ of each cell suspension was added onto LB agar medium and the assay plates were incubated for $24 \mathrm{~h}$. (D) Modified B. glumae flagella assembly downloaded from KEGG and expression profiling of each gene involved in flagella assembly. Each $\log _{2}$ ratio of wild/mutant RPKM value for the flagella assembly gene was transformed to visualize in $\mathrm{MeV}$ (MultiExperiment Viewer), which is downloaded from a public website (http://www.tm4.org/ mev/). 1 $=\log _{2}$ (BGR1/BGS2) before QS onset, 2 $=\log _{2}$ (BGR1/BGS9) before QS onset, 3 $\log _{2}(\mathrm{BGR} 1 / \mathrm{BGS} 2)$ after QS onset, and $4=\log _{2}($ BGR1/BGS9) after QS onset.

onset of QS (Table 4 and Table 5). In addition, all genes (pst $A$, pst $B$, pst $C$, and pst $S$ ) involved in phosphate transporters were strongly up-regulated in a TofI-dependent manner. One of the interesting observations is that most genes (three out of four) involved in RNA polymerase pathways were only up-regulated by TofI before the onset of QS. In addition, several genes (three out of nine) involved in synthesis and degradation of ketone bodies were only up-regulated by Tofl after the onset of QS.

Phenotype analysis of mutants showing QS-dependent gene expression. Because genes involved in motility are the most tightly and positively regulated among all pathways from this study and as several metabolic pathways were strongly regulated by QS, we performed motility assays of mutants that are involved in QS-dependent pathways such as AST pathway and phosphate transport. We selected each gene, astC (bglu_1g10100) and pstA (bglu_1g11470) corresponding to a key gene in the AST pathway and in phosphate transport, respectively. We did not observe any phenotypic differences between BGR1 and pstA mutant strains. However, the ast $C$ mutant showed defects in swarming motility compared with that of wild-type BGR1 (Fig. 5). 
Table 2. Expression profiling of genes involved in the flagellar assembly pathway in B. glumae before and after quorum sensing (QS) onset

\begin{tabular}{|c|c|c|c|c|c|}
\hline \multirow[b]{2}{*}{ Locus ID } & \multirow[b]{2}{*}{ Gene name } & \multicolumn{2}{|c|}{ Before QS onset } & \multicolumn{2}{|c|}{ After QS onset } \\
\hline & & $\begin{array}{c}\log _{2} \\
(\mathrm{R} 1 / \mathrm{S} 2)^{1}\end{array}$ & $\begin{array}{c}\log _{2} \\
(\mathrm{R} 1 / \mathrm{S} 9)^{2}\end{array}$ & $\begin{array}{c}\log _{2} \\
(\mathrm{R} 1 / \mathrm{S} 2) \\
\end{array}$ & $\begin{array}{c}\log _{2} \\
\text { (R1/S9) }\end{array}$ \\
\hline bglu_1g00170 & fliR & 0.9 & 0.2 & 1.4 & -0.1 \\
\hline bglu_1g00180 & fliQ & 1.7 & 0.6 & 2.3 & 1.4 \\
\hline bglu_1g00190 & fliP & 1.5 & 0.9 & 1.9 & 1 \\
\hline bglu_1g00200 & $\mathrm{fliO}$ & 1.2 & 0.6 & 2.3 & 0.8 \\
\hline bglu_2g13030 & $\mathrm{fliC}$ & 0.6 & -0.1 & 2.3 & 1.4 \\
\hline bglu_1g33530 & $f l i G$ & 1.0 & 0.4 & 1.6 & 0.6 \\
\hline bglu_1g00220 & fliM & 2.1 & 0.9 & 2.7 & 1.0 \\
\hline bglu_1g00210 & $f l i N$ & 1.4 & 0.6 & 2.3 & 1.1 \\
\hline bglu_1g01790 & $f l h C$ & 1.0 & 0.3 & 1.3 & 0.2 \\
\hline bglu_1g01780 & $f l h D$ & 1.4 & 0.6 & 1.4 & 0.2 \\
\hline bglu_1g01700 & fliD & 0.6 & 0.3 & 1.8 & 0.5 \\
\hline bglu_1g01940 & $\operatorname{flh} B$ & 1.6 & 0.9 & 2.5 & 0.6 \\
\hline bglu_1g01950 & flhA & 2 & 1.1 & 2.2 & 0.7 \\
\hline bglu_1g01970 & $f l h G$ & 1.8 & 0.8 & 3.3 & 0.9 \\
\hline bglu_1g33060 & $f l g L$ & 0.7 & 0.1 & 1.8 & 0 \\
\hline bglu_1g33070 & $f \lg K$ & 0.9 & 0.3 & 1.7 & 0.3 \\
\hline bglu_1g33100 & flgI & 1.5 & 0.6 & 2.5 & 0.6 \\
\hline bglu_1g33110 & $f l g H$ & 1.8 & 0.7 & 3 & 0.7 \\
\hline bglu_1g33120 & $f \lg G$ & 1.9 & 0.8 & 3 & 0.5 \\
\hline bglu_1g33130 & $f l g F$ & 2 & 0.8 & 3.4 & 0.7 \\
\hline bglu_1g33140 & $f \lg E$ & 1.7 & 0.9 & 3.3 & 0.8 \\
\hline bglu_1g33150 & $f \lg D$ & 2.2 & 1.1 & 3.4 & 0.8 \\
\hline bglu_1g33160 & $f \lg C$ & 2.2 & 1 & 3.6 & 1.1 \\
\hline bglu_1g33170 & $f \lg B$ & 2.1 & 0.9 & 3.4 & 1.2 \\
\hline bglu_1g33180 & $\operatorname{flg} A$ & 1.8 & 0.8 & 4 & 1.1 \\
\hline bglu_1g33190 & $f \lg M$ & 0.3 & 0.1 & 1.3 & 0.4 \\
\hline bglu_1g33200 & $f \lg N$ & 0.2 & 0.3 & 1.1 & 0.2 \\
\hline bglu_1g33490 & $f l i T$ & 0.8 & 0.2 & 2.1 & 0.7 \\
\hline bglu_1g33500 & $f l i S$ & 1 & 0.4 & 1.2 & 0.7 \\
\hline bglu_1g33510 & $f l i E$ & 1.6 & 0.9 & 1.9 & 1 \\
\hline bglu_1g33520 & $f l i F$ & 0.7 & 0.4 & 1.5 & 0.6 \\
\hline bglu_1g33540 & $\mathrm{fliH}$ & 1.1 & 0.6 & 2 & 0.9 \\
\hline bglu_1g33550 & fliI & 1.1 & 0.7 & 1.6 & 0.6 \\
\hline bglu_1g33560 & fliJ & 1.3 & 1 & 2.5 & 0.8 \\
\hline bglu_1g33570 & $f l i K$ & 0.5 & -0.1 & 2 & 0.8 \\
\hline
\end{tabular}

${ }^{1} \log _{2}(\mathrm{R} 1 / \mathrm{S} 2)=\log _{2}(\mathrm{BGR} 1 / \mathrm{BGS} 2)$

${ }^{2} \log _{2}(\mathrm{R} 1 / \mathrm{S} 9)=\log _{2}$ (BGR1/BGS9)

\section{Discussion}

Bacteria can communicate in local population densities through QS. Upon reaching a threshold in signal concentration, gene expression levels undergo changes, leading to a diverse range of behaviors. Although QS-dependent phenotypes differ in each bacterium, motility, biofilm formation, toxin production, and virulence are some of the common features in bacteria (Deng et al., 2011; Goo et al., 2010; Jimenez et al., 2012; Labbate et al., 2004; Milton, 2006; Moreira et al., 2010). In B. glumae, QS-dependent phenotypes were demonstrated by several groups and most of them are well-known characteristics in other bacteria such as toxoflavin production, virulence, detoxification of oxidative stress, and motility. In this study, we analyzed the 
Table 3. Expression profiling of genes involved the in ribosome pathway in B. glumae before and after quorum sensing (QS) onset

\begin{tabular}{|c|c|c|c|c|c|}
\hline \multirow{2}{*}{ Locus ID } & \multirow{2}{*}{ Gene name } & \multicolumn{2}{|c|}{ Before QS onset } & \multicolumn{2}{|c|}{ After QS onset } \\
\hline & & $\log _{2}(\mathrm{R} 1 / \mathrm{S} 2)^{1}$ & $\log _{2}(\mathrm{R} 1 / \mathrm{S} 9)^{2}$ & $\log _{2}(\mathrm{R} 1 / \mathrm{S} 2)$ & $\log _{2}(\mathrm{R} 1 / \mathrm{S} 9)$ \\
\hline bglu 1 g01720 & $r p s U$ & 0.4 & 0.3 & -1.3 & -1.2 \\
\hline bglu_1g02460 & $r p l K$ & 1 & 0.7 & -1.1 & -0.9 \\
\hline bglu 1 g02470 & rpla & 0.8 & 0.4 & -1.1 & -0.9 \\
\hline bglu_1g02480 & rplJ & 1.4 & 0.9 & -1.5 & -1.2 \\
\hline bglu_1g02490 & rplL & 1.3 & 0.7 & -1.5 & -1.3 \\
\hline bglu_1g02530 & $r p s L$ & 1.1 & 0.8 & -1.4 & -1.4 \\
\hline bglu 1 g02540 & $r p s G$ & 1.1 & 0.8 & -1.3 & -1.2 \\
\hline bglu 1 g02570 & $r p s J$ & 1.2 & 0.9 & -1.3 & -1.4 \\
\hline bglu 1 g02580 & rplC & 1.1 & 0.8 & -1.7 & -1.6 \\
\hline bglu 1 g02590 & rplD & 1.3 & 0.9 & -1.7 & -1.8 \\
\hline bglu_1g02600 & rplW & 1.4 & 1.1 & -1.6 & -1.6 \\
\hline bglu 1 g02610 & $r p l B$ & 1.3 & 0.9 & -1.5 & -1.5 \\
\hline bglu_lg02620 & $\operatorname{rps} S$ & 1.5 & 1 & -1.5 & -1.4 \\
\hline bglu_1g02630 & rplV & 1.4 & 0.9 & -1.6 & -1.5 \\
\hline bglu_1g02640 & $r p s C$ & 1.4 & 1 & -1.6 & -1.3 \\
\hline bglu_1g02650 & rplP & 1.5 & 1.1 & -1.5 & -1.3 \\
\hline bglu_lg02660 & rpmC & 1.4 & 1 & -1.7 & -1.4 \\
\hline bglu 1 g02670 & $r p s Q$ & 1.3 & 0.9 & -1.4 & -1.3 \\
\hline bglu 1 g02680 & rplN & 1.3 & 0.9 & -1.7 & -1.9 \\
\hline bglu $1 \mathrm{~g} 02690$ & $\operatorname{rpl} X$ & 1.1 & 0.8 & -1.5 & -1.7 \\
\hline bglu 1 g02700 & rple & 1.2 & 0.8 & -1.4 & -1.7 \\
\hline bglu_1g02710 & $r p s N$ & 1.4 & 1 & -1.6 & -1.7 \\
\hline bglu_1g02720 & $r p s H$ & 1.2 & 0.8 & -1.6 & -1.7 \\
\hline bglu_1g02730 & $r p l F$ & 1.4 & 0.9 & -1.4 & -1.5 \\
\hline bglu_1g02740 & rplR & 1.3 & 0.8 & -1.3 & -1.5 \\
\hline bglu_1g02750 & $r p s E$ & 1.2 & 0.8 & -1.2 & -1.2 \\
\hline bglu_1g02760 & $\operatorname{rpmD}$ & 1.3 & 0.7 & -1.1 & -1 \\
\hline bglu_1g02770 & rplO & 1.2 & 0.6 & -1.3 & -1.3 \\
\hline bglu_1g02800 & rpmJ & 1.3 & 1 & -1.3 & -1.4 \\
\hline bglu 1 g02810 & rps $M$ & 1.1 & 0.7 & -1.4 & -1.4 \\
\hline bglu 1 g02820 & $\operatorname{rps} K$ & 1.1 & 0.8 & -1.3 & -1.2 \\
\hline bglu 1 g02830 & $\operatorname{rps} D$ & 1.1 & 0.7 & -1 & -1.2 \\
\hline bglu 1 g02850 & $r p l Q$ & 0.8 & 0.4 & -1.3 & -1.3 \\
\hline bglu_1g04830 & rplu & 1.1 & 0.8 & -1.3 & -0.9 \\
\hline bglu_lg04840 & гртA & 1 & 0.7 & -1 & -0.9 \\
\hline bglu_lg05690 & rplM & 1.3 & 1 & -1.8 & -1.4 \\
\hline bglu_lg05700 & rpsI & 1 & 0.7 & -1.4 & -1.4 \\
\hline bglu 1 g088800 & $\operatorname{rps} A$ & 0.7 & 0.5 & -1.1 & -1.2 \\
\hline bglu_1g09030 & $r p s P$ & 0.6 & 0.4 & -1.4 & -0.9 \\
\hline bglu_1g09060 & rpls & 0.7 & 0.6 & -1.4 & -1.1 \\
\hline bglu 1 g09480 & $r p m F$ & 0.4 & 0.5 & -1 & -0.6 \\
\hline bglu 1 g12720 & $r p s B$ & 1.2 & 0.8 & -1.3 & -1.3 \\
\hline bglu 1g13940 & $r p s F$ & 1 & 0.6 & -1.1 & -0.9 \\
\hline bglu 1 g13960 & $\operatorname{rps} R$ & 1 & 0.6 & -1.1 & -1.3 \\
\hline bglu lg13970 & rplI & 0.8 & 0.5 & -0.9 & -1.1 \\
\hline bglu_1g14290 & rpmE2 & 0.8 & 0.6 & -2 & -1.4 \\
\hline bglu_lg18160 & $r p s U$ & -1 & -1.5 & -0.2 & -0.5 \\
\hline bglu_lg21490 & rplT & 1 & 0.8 & -1.4 & -1.1 \\
\hline bglu_1g21500 & rpmI & 1.3 & 0.9 & -1.3 & -1.2 \\
\hline bglu_lg25990 & rpsO & 0.6 & 0.5 & -1.5 & -1.6 \\
\hline bglu_1g28680 & rpmB & 0.7 & 0.6 & -1.8 & -1 \\
\hline bglu_1g28690 & rpmG & 0.5 & 0.4 & -1.5 & -1.1 \\
\hline bglu_lg29160 & $r p s T$ & 0.9 & 0.8 & -1.8 & -1.4 \\
\hline bglu lg31330 & rplY & 1.1 & 0.7 & -0.8 & -0.9 \\
\hline bglu_2g15900 & $r p s U$ & 0.5 & 0 & -1.5 & -1.1 \\
\hline
\end{tabular}


Table 4. Expression profiling of genes involved in the arginine degradation II pathway in B. glumae before and after quorum sensing (QS) onset

\begin{tabular}{cccccc}
\hline \hline \multirow{2}{*}{ Locus ID } & \multirow{2}{*}{ Gene name } & \multicolumn{2}{c}{ Before QS onset } & \multicolumn{2}{c}{ After QS onset } \\
\cline { 3 - 6 } \cline { 3 - 5 } & & $\log _{2}(\mathrm{R} 1 / \mathrm{S} 2)^{1}$ & $\log _{2}(\mathrm{R} 1 / \mathrm{S} 9)^{2}$ & $\log _{2}(\mathrm{R} 1 / \mathrm{S} 2)$ & 0.9 \\
bglu_1g10100 $_{\text {argD/astC }}$ & ast $A$ & -1.2 & -0.9 & 0.8 & 0.8 \\
bglu_1g10110 & ast $A$ & -1.1 & -0.8 & 0.9 & 0.9 \\
bglu_1g10120 & astD & -1 & -0.5 & 0.8 & 0.9 \\
bglu_1g10130 & astB & -0.9 & -0.6 & 0.6 & 0.8 \\
bglu_1g10140 & astE & -1.1 & -0.6 & 0.8 & 0.8 \\
bglu_1g10150 & & -0.7 & 0.8
\end{tabular}

${ }^{1} \log _{2}(\mathrm{R} 1 / \mathrm{S} 2)=\log _{2}(\mathrm{BGR} 1 / \mathrm{BGS} 2)$

${ }^{2} \log _{2}(\mathrm{R} 1 / \mathrm{S} 9)=\log _{2}($ BGR1/BGS9)

Table 5. Expression profiling of several key genes involved in the fatty acid $\beta$-oxidation I pathway in B. glumae before and after quorum sensing (QS) onset

\begin{tabular}{|c|c|c|c|c|c|}
\hline \multirow{2}{*}{ Locus ID } & \multirow{2}{*}{ Gene name } & \multicolumn{2}{|c|}{ Before QS onset } & \multicolumn{2}{|c|}{ After QS onset } \\
\hline & & $\log _{2}(\mathrm{R} 1 / \mathrm{S} 2)$ & $\log _{2}(\mathrm{R} 1 / \mathrm{S} 9)$ & $\log _{2}(\mathrm{R} 1 / \mathrm{S} 2)$ & $\log _{2}(\mathrm{R} 1 / \mathrm{S} 9)$ \\
\hline bglu_2g06260 & $f a d E$ & -0.4 & -0.6 & 2.1 & 1.7 \\
\hline bglu_2g06270 & fadD & -0.4 & -0.6 & 1.5 & 0.9 \\
\hline bglu_2g06300 & fadB/fadJ & -0.7 & -0.5 & 1.6 & 0.9 \\
\hline bglu_2g06310 & $\mathrm{fadB}$ & -0.4 & -0.7 & 1.3 & 0.4 \\
\hline
\end{tabular}

${ }^{1} \log _{2}(\mathrm{R} 1 / \mathrm{S} 2)=\log _{2}($ BGR1/BGS2)

${ }^{2} \log _{2}(\mathrm{R} 1 / \mathrm{S} 9)=\log _{2}(\mathrm{BGR} 1 / \mathrm{BGS} 9)$

profiling of QS-dependent gene expression using two QS mutants at two different time points (before and after the onset of QS) to understand QS-dependent phenotypes and signaling pathways through a combination of RNAseqbased differential expressed genes (DEGs) with GO enrichment and pathway analyses. Through this approach, we found that QS-regulated genes are not only involved in several metabolic pathways but also our results are consistent with QS-dependent phenotypes such as motility.

In QS-dependent metabolic pathways, we observed a couple of instances of amino acid degradation and changes of carbon catabolism. Particularly, all genes involved in arginine degradation II and phosphate transport were up-regulated after the onset of QS (Table 2 and Table 4). In addition, several key genes involved in fatty acid $\beta$-oxidation I were also up-regulated in a QS-dependent manner (Table 5). Thus, QS can induce changes of gene expression in carbon and nitrogen catabolism as well as fatty acid degradation in response to high bacterial density.

Interestingly, all genes in ribosome pathway are downregulated after the onset of QS, whereas these genes are upregulated before the onset of QS. Although how these genes involved in ribosome pathway are tightly regulated by QS is not known, it is partly consistent with the observation that QS mutants grow faster than B. glumae BGR1 during culture stages (data not shown). It may be caused by the disruption of recognition in high bacterial density in mutants, whereas the wild-type bacteria can recognize it and modify cell metabolisms to withstand negative effects such as limited food sources and toxic wastes caused by high densities.

For understanding of the putative function of DEGs from RNAseq analysis, we employed GO enrichment instead of simple GO counting. This GO enrichment can provide statistical reliability on the observed GO for differentially expressed genes. Tofl-dependent genes exhibited the highest enrichment of motility-related terms in all three GOs. For example, flagellar motility for GO biological process, motor activity for GO molecular function, and flagellum for GO cellular component were the highest enriched in TofIregulated genes. This observation indicates that genes involved in flagellar motility were strongly regulated by TofI, which is consistent with the B. glumae QS-dependent phenotype such as swarming motility. In particular, all genes involved in flagellar assembly and motility were upregulated 2-fold to 16-fold in a Tofl-dependent manner. For common GO between Tofl- and QsmR-dependent genes, DNA replication and medication were highly enriched.

As far as QS-dependent phenotypes are concerned, motility-related genes among all the observed pathways are the most tightly regulated by TofI. As a previous report (Kim et al., 2007), QsmR regulated by Tofl and sub- 
A
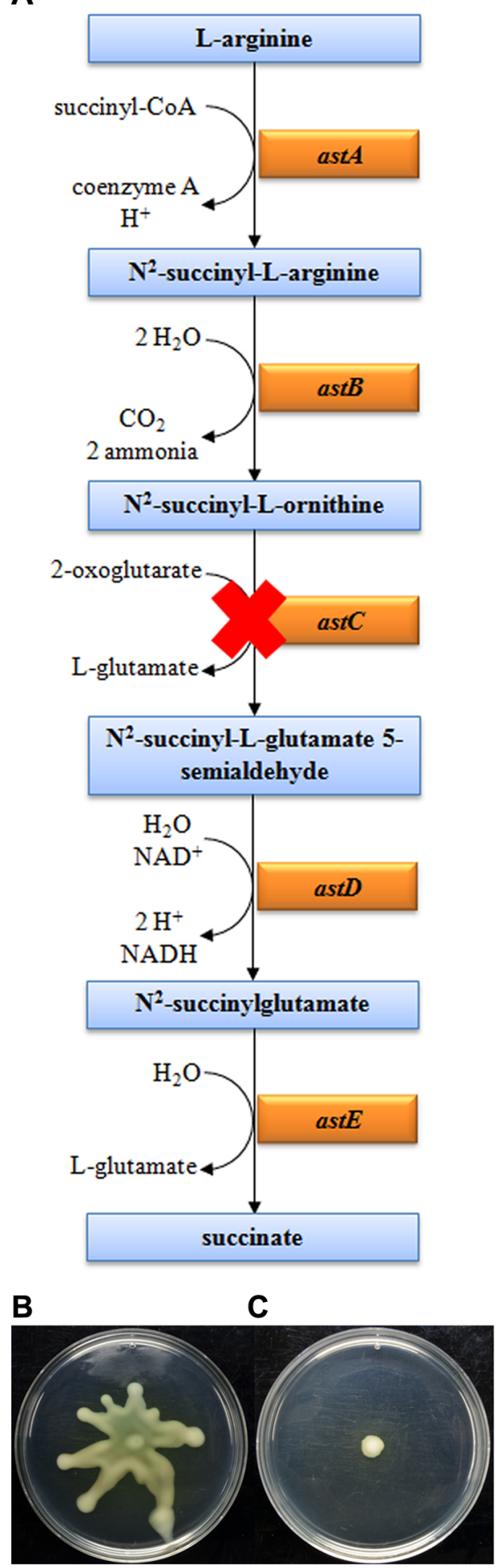

Fig. 5. Arginine degradation II (AST pathway) genes and phenotype assays of astC mutant in AST pathway. (A) Modified AST pathway downloaded from BioCyc $(B, C)$ Swarming motility assays on wild-type and $a s t C$ mutant, respectively. An aliquot (2 $\mu \mathrm{l}$ ) of each cell suspension was added to LB agar medium and the assay plates were incubated for $24 \mathrm{~h}$. sequently by $f l h D C$ was activated by QsmR. FlhDC in turn activated the expression of genes involved in flagellum biosynthesis and motor functions. However, in this study we found that Tofl strongly controlled the up-regulation of genes involved in flagellum biosynthesis and motor functions but QsmR did not (Table 2). In addition, swarming motility assays showed complete defects in BGS2, whereas in BGS9 slight swarming defects showed up a few times whenever these two mutants were used as controls for swarming tests of other mutants. This implies that Tofl can regulate motility not only in a QsmR-dependent manner but also through unknown regulators or pathways. Thus, the phenotype of BGS2 can exhibit complete defects in motility.

Following reports on the significance of metabolic pathway shifts caused by QS (Goo et al., 2012; Pai et al., 2012), we investigated QS-dependent metabolic pathways by combination of DEGs with pathway analysis from KEGG or Biocyc. We observed three positively regulated pathways including arginine degradation II, phosphate transport, and fatty acid $\beta$-oxidation I. To study the relationship between these pathways and QS signaling cascade, mutants for these pathways and assayed mutant phenotypes were generated. Two mutants, ast $C$-mutant-related arginine degradation II and fadE (bglu_2g06260) mutant, involved in fatty acid $\beta$ oxidation I showed defects in swarming motility, whereas pstA mutant involved in phosphate transport did not show any phenotypes compared with wild type. The ast $C$ mutant exhibited strong defects in swarming motility similar to BGS9 (Fig. 5) and the fadE mutant reduced swarming motility compared with the wild type (data not shown). These mutants may be indirectly related to flagella motility because mutants of key regulators (e.g., $f l h C$ and $f l h D$ ) in flagella motility are observed to change through several metabolic pathways (Kapatral et al., 2004). Thus, we suggest that QS-dependent metabolic pathways can directly or indirectly regulate bacteria motility. Future work is needed to identify relationships between QS-dependent motility and metabolic pathways through in-depth studies of these mutants involved in metabolic pathways.

In conclusion, we observed QS-dependent GO enrichment or pathway including DNA modification in both TofI- and QsmR-dependent genes. The genes involved in flagellar motility and ribosome pathways were up-regulated and down-regulated in a QS-dependent manner, particularly by TofI. The reported phenotypes as well as phenotypic validation from this study were consistent with the QS-dependent pathways and GO enrichments. Integration of GO enrichment or metabolic pathways is useful in application of generated DEGs from B. glumae QS-dependent RNAseq analysis to understand cellular, molecular, and metabolic phenomena before and after the onset of QS. 


\section{Acknowledgments}

This work was supported by a 2-year Research Grant of Pusan National University (Y.-S. S.).

\section{References}

Cother, E. J., Noble, D. H., de Ven, R. J. van., Lanoiselet, V., Ash, G., Vuthy, N., Visarto, P. and Stodart, B. 2010. Bacterial pathogens of rice in the Kingdom of Cambodia and description of a new pathogen causing a serious sheath rot disease. Plant Pathol. 5:944-953.

Chun, H., Choi, O., Goo, E., Kim, N., Kim, H., Kang, Y., Kim, J., Moon, J. S. and Hwang, I. 2009. The quorum sensing-dependent gene katG of Burkholderia glumae is important for protection from visible light. J. Bacteriol. 191:4152-4157.

Deng, Y., Wu, J., Tao, F. and Zhang, L. H. 2011. Listening to a new language: DSF-based quorum sensing in Gram-negative bacteria. Chem. Rev. 111:160-173.

Devescovi, G., Bigirimana, J., Degrassi, G., Cabrio, L., LiPuma, J. J., Kim, J., Hwang, I. and Venturi, V. 2007. Involvement of a quorum-sensing-regulated lipase secreted by a clinical isolate of Burkholderia glumae in severe disease symptoms in rice. Appl. Environ. Microbiol. 73:4950-4958.

Fuqua, W. C., Winans, S. C. and Greenberg, E. P. 1994. Quorum sensing in bacteria: The LuxR-LuxI family of cell densityresponsive transcriptional regulators. J. Bacteriol. 176:269275.

Goo, E., Majerczyk, C. D., An, J. H., Chandler, J. R., Seo, Y.-S., Ham, H., Lim, J. Y., Kim, H., Lee, B., Jang, M. S., Greenberg, E. P. and Hwang, I. 2012. Bacterial quorum sensing, cooperativity, and anticipation of stationary-phase stress. Proc. Natl. Acad. Sci. USA 109:19775-19780.

Jimenez, P. N., Koch, G., Thompson, J. A., Xavier, K. B., Cool, R. H. and Quax, W. J. 2012. The multiple signaling systems regulating virulence in Pseudomonas aeruginosa. Microbiol. Mol. Biol. Rev. 76:46-65.

Kapatral, V., Campbell, J. W., Minnich, S. A., Thomson, N. R., Matsumura, P. and Prüss, B. M. 2004. Gene array analysis of Yersinia enterocolitica FlhD and FlhC: regulation of enzymes affecting synthesis and degradation of carbamoylphosphate. Microbiology 150:2289-2300.

Kim, J., Kim, J. G., Kang, Y., Jang, J. Y., Jog, G. J., Lim, J. Y., Kim, S., Suga, H., Nagamatsu, T. and Hwang, I. 2004. Quorum sensing and the LysR-type transcriptional activator ToxR regulate toxoflavin biosynthesis and transport in Burkholderia glumae. Mol. Microbiol. 54:921-934.

Kim, J., Kang, Y., Choi, O., Jeong, Y., Jeong, J. E., Lim, J. Y., Kim, M., Moon, J. S., Suga, H. and Hwang, I. 2007. Regulation of polar flagellum genes is mediated by quorum sensing and FlhDC in Burkholderia glumae. Mol. Microbiol. 64:165179.

Kim, J., Oh, J., Choi, O., Kang, Y., Kim, H., Goo, E., Ma, J., Nagamatsu, T., Moon, J. S. and Hwang, I. 2009. Biochemical evidence for ToxR and ToxJ binding to the tox operons of Burkholderia glumae and mutational analysis of ToxR. J. Bacteriol. 191:4870-4878.

Kim, J., Kang, Y., Kim, J.-G., Choi, O. and Hwang, I. 2010. Occurrence of Burkholderia glumae on rice and field crops in Korea. Plant Pathol. J. 26:271-272.

Labbate, M., Queck, S. Y., Koh, K. S., Rice, S. A., Givskov, M. and Kjelleberg, S. 2004. Quorum sensing-controlled biofilm development in Serratia liquefaciens MG1. J. Bacteriol. 186:692-698.

Milton, D. L. 2006. Quorum sensing in vibrios: complexity for diversification. Int. J. Med. Microbiol. 296:61-71.

Moreira, C. G., Weinshenker, D. and Sperandio, V. 2010. QseC mediates Salmonella enterica serovar typhimurium virulence in vitro and in vivo. Infect. Immun. 78:914-926.

Mortazavi, A., Williams, B. A., McCue, K., Schaeffer, L. and Wold, B. 2008. Mapping and quantifying mammalian transcriptomes by RNA-Seq. Nat. Methods 5:621-628.

Nandakumar, R., Shahjahan, A. K. M., Yuan, X. L., Dickstein, E. R., Groth, D. E., Clark, C. A., Cartwright, R. D. and Rush, M. C. 2009. Burkholderia glumae and B. gladioli cause bacterial panicle blight in rice in the southern United States. Plant Dis. 93:896-905.

Pai, A., Tanouchi, Y. and You, L. 2012. Optimality and robustness in quorum sensing (QS)-mediated regulation of a costly public good enzyme. Proc. Natl. Acad. Sci. USA 109:19810-19815.

Sambrook, J., Fritsch, E. F. and Maniatis, T. 1989. Molecular cloning: a laboratory manual, $2^{\text {nd }}$ ed. Cold Spring Harbor laboratory Press, Cold Srping Harbor, NY.

Sato, Z., Koiso, Y., Iwasaki, S. and Matsuda, I. 1989. Toxins produced by Pseudomonas glumae. Ann. Phytopathol. Soc. Jpn. 55:356-359.

Storey, J. D. and Tibshirani, R. 2003. Statistical significance for genomewide studies. Proc. Natl. Acad. Sci. USA. 100:94409445 . 